\title{
Institutionnalisation de la recherche et formation des ingénieurs à Grenoble (années 1960-1980)
}

Engineering Education and Institutionalization of Research (1960-1980)

\section{Thomas Lerosier}

\section{OpenEdition}

Journals

Édition électronique

URL : https://journals.openedition.org/histoire-education/4696

DOI : 10.4000/histoire-education.4696

ISSN : 2102-5452

Éditeur

ENS Éditions

\section{Édition imprimée}

Date de publication : 31 décembre 2019

Pagination : 87-110

ISBN : 979-10-362-0144-8

ISSN : 0221-6280

Référence électronique

Thomas Lerosier, «Institutionnalisation de la recherche et formation des ingénieurs à Grenoble (années 1960-1980) », Histoire de l'éducation [En ligne], 152 | 2019, mis en ligne le 01 janvier 2022, consulté le 06 janvier 2022. URL : http://journals.openedition.org/histoire-education/4696 ; DOI : https://doi.org/10.4000/histoire-education.4696 


\title{
Institutionnalisation de la recherche et formation des ingénieurs à Grenoble (années 1960-1980)
}

\author{
Thomas Lerosier
}

Depuis leur création jusqu'à leurs évolutions les plus récentes, les institutions de recherche et d'enseignement supérieur sont intégrées à des contextes scientifiques, économiques et politiques locaux ${ }^{1}$. En particulier, à partir des années 1880 , les facultés des sciences de province commencent à se doter, à l'initiative d'universitaires locaux, d'instituts techniques destinés à former les techniciens et les ingénieurs dont l'industrie, tant régionale que nationale, a besoin ${ }^{2}$. Les villes de Nancy, Toulouse et Grenoble sont les premières à se doter de tels instituts. Ces créations initient la formation d'ingénieurs dans les villes de province en France ${ }^{3}$. Mais à compter de la Seconde Guerre mondiale, une plus grande centralisation des affaires scientifiques et technologiques est conduite par l'État avec la mise en œuvre de grandes politiques nationales de développement technologique et d'aménagement du territoire ${ }^{4}$. La création du Centre national pour la recherche scientifique (CNRS) en 1939, la création des

1 Laurent Rollet, "Peut-on faire l'histoire des pôles scientifiques?", Histoire de l'éducation, $\mathrm{n}^{\circ} 122$, 2009, p. 93-113.

2 Robert Fox, George Weisz (dir.), The Organization of Science and Technology in France, 1808-1914, Cambridge/Paris, Cambridge University Press/Éditions de la Maison des sciences de l'Homme, 1980.

3 Laurent Rollet, "Peut-on faire l'histoire des pôles scientifiques?", art. cit., p. 96.

4 Voir le chapitre "1950-1968 : le retour au centralisme", dans Michel Grossetti et al., Villes et institutions scientifiques, Programme interdisciplinaire de recherche sur les villes (PIR-VILLES - CNRS), Région Midi-Pyrénées, 1996, p. 316. 
grands organismes dans le domaine des télécommunications (CNET) en 1944, de l'énergie atomique (CEA) en 1945 et de l'agriculture (INRA) en 1946 ainsi que la transformation des instituts techniques en "écoles nationales supérieures d'ingénieurs" (ENSI) en sont les conséquences directes.

À partir du cas de l'Institut polytechnique de Grenoble, nous proposons ici de revenir sur la manière dont les évolutions des institutions d'enseignement supérieur et de recherche sont modelées, depuis les années soixante, à la fois par des configurations locales et par les enjeux nationaux.

La question des configurations locales a fait l'objet d'un certain nombre d'analyses de la part des historiens des sciences et de l'éducation. Les évolutions institutionnelles provinciales sont profondément inscrites dans des contextes locaux ${ }^{6}$. Par exemple, l'ouvrage dirigé par André Grelon et Françoise Birck, Des ingénieurs pour la Lorraine, montre les effets de la structure industrielle sur la formation technique ${ }^{7}$. Le contexte économique et industriel joue un rôle central dans la création d'instituts techniques. Cependant, l'attention portée aux configurations locales ne doit pas conduire à sous-estimer la dynamique nationale du système d'enseignement supérieur français et ses conséquences sur les processus locaux. Le cas toulousain, abondamment documenté par Michel Grossetti et ses co-auteurs, montre que les évolutions structurelles et institutionnelles d'un système scientifique local se calquent la plupart du temps sur celles du système national ${ }^{8}$. Leurs divergences - lorsqu'il y en a correspondent bien souvent à des décalages dans le temps en raison d'inerties locales. La problématique des pôles scientifiques est donc au cœur d'un jeu d'échelles entre évolutions structurelles de l'enseignement supérieur et de la recherche, politiques nationales et contingences locales ${ }^{9}$.

5 Par convention et malgré les nombreuses évolutions institutionnelles que l'Institut polytechnique de Grenoble a connues depuis sa création en 1910, nous parlerons de "l'Institut" tout au long de cet article. Nous nous référons aux dénominations institutionnelles exactes lorsque cela s'avère nécessaire à notre démonstration.

6 Renaud d'Enfert, "Circulations mathématiques et offre locale d'enseignement : le cas de Troyes sous la Restauration et la monarchie de Juillet", Philosophia Scientiæ, vol. 19, n², 2015, p. 79-94; Philippe Savoie, "Offre locale et engagement de l'État. Les enseignements technique et primaire supérieur à Nancy et les conditions de leur évolution sous la Troisième République", Histoire de l'éducation, $\mathrm{n}^{\circ} 66,1995$, p. 47-83.

7 Françoise Birck, André Grelon (dir.), Des ingénieurs pour la Lorraine $\mathrm{XIX}^{e}-\mathrm{XX} \mathrm{X}^{e}$ siècles, Metz, Éditions Serpenoise, 1998.

8 Michel Grossetti (dir.), Université et territoire : un système local d'enseignement supérieur Toulouse et Midi-Pyrénées, Toulouse, Presses universitaires du Mirail, 1994, p. 228.

9 Laurent Rollet, "Peut-on faire l'histoire des pôles scientifiques? ", art. cit. 
Par ailleurs, la focale sur les configurations locales en matière d'enseignement technique ne doit pas conduire non plus à idéaliser les rapports entre science et industrie. L'historiographie des villes scientifiques a montré que la relation entre la science académique et le monde industriel est un processus central dans la structuration de la recherche contemporaine, mais qu'il n'a rien d'évident ${ }^{10}$. Contre l'illusion d'un "mariage de la science et de l'industrie" (qu'on trouvera abondamment dans les discours publics), certaines recherches ont mis en lumière que leur rapprochement a été un processus long et difficile ${ }^{11}$. Dominique Pestre, François Jacq et François Caron ont souligné ce phénomène dans le cas de Grenoble en montrant qu'avant les années soixante-dix, les rapports entre les industriels et les physiciens grenoblois sont encore relativement ponctuels ${ }^{12}$. André Grelon et Françoise Birck le soulignent également dans le cas de $\mathrm{Nancy}^{13}$. Le rapport Villes et institutions scientifiques nuance l'idée selon laquelle les industriels locaux auraient été au cœur du développement des instituts techniques des facultés des sciences. Il montre, au contraire, que le "projet d'un développement économique fondé sur la science est d'abord celui des universitaires $" 14$.

L'Institut polytechnique de Grenoble est un cas d'étude particulièrement pertinent lorsqu'on s'intéresse à la relation entre l'enseignement supérieur et la recherche scientifique. En effet, Grenoble est devenue, au début des années 1990, le "plus grand pôle de recherche de province" grâce à la physique et aux sciences de l'ingénieur ${ }^{15}$, la ville se caractérisant essentiellement par une

10 Françoise Birck, Marie-Jeanne Choffel-Mailfert, "Introduction", in Laurent Rollet, Marie-Jeanne Choffel-Mailfert (dir.), Aux origines d'un pôle scientifique : faculté des sciences et écoles d'ingénieurs à Nancy du Second Empire aux années 1960, Nancy, Presses universitaires de Nancy, 2007, p. 10-11.

11 Françoise Birck, "Introduction : la Lorraine et ses enseignements techniques supérieurs ", in Françoise Birck, André Grelon (dir.), Des ingénieurs pour la Lorraine..., op. cit., p. 23.

12 Dominique Pestre, Louis Néel, le magnétisme et Grenoble : récit de la création d'un empire physicien dans la province française 1940-1965, Paris, Éditions du CNRS, 1990; François Jacq, Un regard sur la science et l'industrie dans la France d'après-guerre : le cas du laboratoire d'électronique et de technologie de l'informatique (LETI), mémoire de DEA, Paris, École des hautes études en sciences sociales/École nationale supérieure des Mines de Paris, 1991; François Caron, "Le dialogue entre la science et l'industrie à Grenoble. La science et l'industrie, un mariage de raison", La revue pour l'histoire du CNRS, 2000, nº 2, p. 44-52; Éric Robert, L'ingénieur, moteur de l'innovation : un siècle de formation d'ingénieurs à Grenoble, Grenoble, Éditions des Vignes/l'Éditeur/Institut national polytechnique de Grenoble, 2001.

13 Françoise Birck, André Grelon (dir.), Des ingénieurs pour la Lorraine..., op. cit., p. 23.

14 Michel Grossetti et al., Villes et institutions scientifiques, op. cit., p. 316.

15 Michel Grossetti, Science, industrie et territoire, Toulouse, Presses universitaires du Mirail, 1995 , p. 107. 
concentration importante de chercheurs du CNRS et par la présence de grands établissements comme le Commissariat à l'énergie atomique (CEA). L'étude du cas grenoblois peut en outre contribuer à pallier le manque signalé de travaux d'histoire de l'enseignement supérieur portant sur la période allant de 1968 aux années $1990^{16}$. De fait, les travaux sur l'enseignement technique supérieur en province évoqués précédemment se sont essentiellement concentrés sur le $\mathrm{XIX}^{\mathrm{e}}$ siècle et les deux premiers tiers du XX $\mathrm{X}^{\mathrm{e}}$. Le présent article s'attache donc à poursuivre leurs efforts en s'intéressant aux évolutions de l'enseignement supérieur postérieures à la réforme Edgar Faure de 1968.

Cet article est principalement basé sur l'analyse des archives de l'Institut national polytechnique de Grenoble (INPG). Portant sur la période 1940-1990, celles-ci sont déposées aux archives départementales de l'Isère. Les documents concernant les décennies 1950 à 1970 se sont révélés les plus riches et les plus complets. Plus précisément, le présent article s'appuie sur le traitement des documents relatifs aux principaux organes de l'Institut : le conseil de perfectionnement et le conseil scientifique qui le remplace à partir de 1968, ainsi que, dans une moindre mesure, le conseil d'administration. Le conseil de perfectionnement a pour objet d'émettre des avis sur le contenu des formations, le choix des enseignements ou la création de nouvelles sections. Mais, en pratique, la plupart des enjeux stratégiques de l'Institut y sont abordés. Le conseil scientifique contribue quant à lui à la gouvernance de l'école en matière de politique scientifique et de partenariats de recherche (publics et privés). Ces archives permettent de connaître les préoccupations des membres de l'Institut sur le long terme, de suivre les débats structurants et de se focaliser sur la politique générale de l'institution. Elles ont toutefois deux défauts d'importance. D'abord, elles ne permettent pas de traiter quantitativement la question centrale des financements. Ensuite, elles circonscrivent l'analyse à l'institution elle-même et ne donnent pas accès directement aux stratégies des autres acteurs (industriels, collectivités locales). Nous avons pu contourner en partie ces difficultés, car les acteurs économiques sont présents dans les conseils étudiés et s'y expriment largement.

En définitive, il devient possible de montrer comment, au tournant des années 1970, la recherche scientifique s'affirme comme l'enjeu principal de

16 Emmanuelle Picard, "L'histoire de l'enseignement supérieur français. Pour une approche globale", Histoire de l'éducation, $\mathrm{n}^{\circ}$ 122, 2009, p.30-31. 
la politique de développement de l'Institut. En décrivant comment ce processus est façonné à la fois par des évolutions nationales et des contingences locales, nous mettons à jour un changement d'échelle dans la manière dont se déterminent les priorités au sein de l'établissement. Nous montrons que, s'ils ne disparaissent pas, les enjeux locaux sont profondément reconfigurés par les politiques scientifiques, le pilotage de la recherche et l'évolution des pratiques scientifiques. À partir de cette étude de cas, nous entendons caractériser un processus structurel plus général identifié par Yves Gingras dans le contexte canadien sous l'expression "d'institutionnalisation de la recherche" ${ }^{17}$. S'intéresser aux différentes dimensions (institutionnelle, industrielle, politique) de ce processus et au jeu des échelles locale et nationale implique de tenir ensemble différentes temporalités. C'est cette attention au caractère processuel des évolutions institutionnelles qui nous a conduit, malgré la variation de la qualité des archives disponibles selon les périodes, à adopter une périodisation assez large recouvrant un peu plus de trois décennies et centrée sur les années 1970.

La première partie de l'article décrit l'implication des industriels grenoblois dans la formation des ingénieurs avant 1968. Elle met en lumière l'appariement entre les formations proposées à l'Institut et la structure du milieu industriel grenoblois. À ce moment, l'Institut se préoccupe peu de recherche scientifique et celle-ci, à la manière de la formation, se construit essentiellement en résonance avec le monde industriel. La deuxième partie s'attache à décrire la manière dont les cadres de l'Institut s'emparent de la réforme de l'enseignement supérieur initiée par Edgar Faure pour faire de leur établissement une grande école et le sortir du giron de l'université. La troisième partie de l'article montre que cette évolution introduit un retrait des industriels de la formation des ingénieurs dans un double contexte de mutation industrielle locale et de promotion de la recherche scientifique à l'échelle nationale. Le point de départ de l'institutionnalisation de la recherche correspond à l'adoption par l'Institut d'une politique scientifique explicite. Contrainte par la promotion de la pluridisciplinarité ou les modes de financement de la recherche, cette institutionnalisation repose finalement sur l'intégration des recherches académiques dans les enjeux économiques et technologiques grenoblois. Ce faisant, les laboratoires deviennent,

17 Yves Gingras, "L'institutionnalisation de la recherche en milieu universitaire et ses effets ", Sociologie et sociétés, vol.23, nº1, 1991, p. 39. 
à l'échelle de l'Institut, les nouveaux interlocuteurs du monde industriel et les cadres de l'Institut entreprennent d'adosser la formation des ingénieurs, et toute son identité, non plus au contexte industriel grenoblois, mais à la recherche et aux laboratoires.

\section{Une école d'ingénieurs en phase avec son contexte industriel}

Durant les décennies 1950 et 1960, Grenoble est sans aucun doute l'un des "grands foyers industriels" français ${ }^{18}$. D’un point de vue économique, les années d'après-guerre sont marquées par au moins trois caractéristiques. D’abord, bien qu'en perte de vitesse, l'industrie traditionnelle grenobloise - ganterie, industrie alimentaire, etc. - est encore présente. Ensuite, l'héritage de l'industrialisation liée à la fabrication d'équipements pour la production d'énergie hydroélectrique se maintient. Enfin, de nouvelles activités sont liées au développement de la recherche et des institutions d'enseignement supérieur comme la métallurgie des poudres, le matériel de travaux publics et l'électronique. En 1955, le groupe Thomson crée la CES Cosem, une petite société d'appareillage électrique, et IBM commence à investir dans la région grenobloise ${ }^{19}$.

\section{Les accointances entre formation des ingénieurs et monde industriel}

L'Institut polytechnique de Grenoble est créé en 1910 suite à la progressive mise en place d'un service d'hydraulique et d'un autre d'électrochimie au sein de l'Institut d'électrotechnique de Grenoble (institut technique fondé en 1898). Par le décret du 27 mars 1948, il obtient le statut d'ENSI (école nationale supérieure d'ingénieurs) et devient l'École nationale supérieure d'électrotechnique et d'hydraulique de Grenoble (ENSEHG) ${ }^{20}$. À ce moment et depuis sa création, l'Institut est une composante de l'université de Grenoble, administrativement distincte de la faculté des sciences. Le statut d'ENSI apporte son lot de contraintes en matière de recrutement des élèves et dans le choix des programmes, mais il apporte surtout des financements substantiels qui permettent d'envisager le

18 Gilbert Armand, Villes, centres et organisation urbaine des Alpes du Nord : le passé et le présent, Grenoble, Imprimerie Allier, 1974, p. 369.

19 Henri Morsel, Jean-François Parent, Les industries de la région grenobloise : itinéraire historique et géographique, Grenoble, Presses universitaires de Grenoble, 1991, p. 173.

20 Éric Robert, L'ingénieur, moteur de l'innovation, op. cit., p.67-68. 
développement de l'établissement ${ }^{21}$. L'Institut comprend alors trois sections. La section d'électrotechnique familiarise ses élèves aux méthodes d'utilisation et de transport de l'énergie électrique. La section d'hydraulique prépare les siens à l'utilisation des liquides (énergie hydraulique, génie maritime). La section hautes fréquences forme des ingénieurs spécialisés dans le domaine des ondes électromagnétiques (radionavigation, radars, télécommunications).

Durant la période 1945-1968, la formation des ingénieurs constitue l'activité principale de l'Institut et la recherche y est une activité très secondaire. Son directeur, Louis Néel, est également directeur du laboratoire des essais mécaniques de l'Institut ainsi que du Laboratoire d'électrostatique et de physique du métal (LEPM) de la faculté des sciences. Il est néanmoins très attaché à la formation d'ingénieurs et au développement industriel ${ }^{22}$. La relation entre l'Institut et l'environnement industriel grenoblois est, à ce moment, très concrète : un tiers des membres de son conseil de perfectionnement est issu du monde économique ${ }^{23}$. La présence des industriels témoigne de sa vocation : former des ingénieurs pour les industries (principalement locales) et pour les grands services publics. La présence des représentants du monde industriel rend possible l'identification des besoins des acteurs économiques. Les industries électriques et mécaniques embauchent plus volontiers des ingénieurs formés à l'utilisation des technologies industrielles et immédiatement opérationnels. Les petites industries recrutent surtout localement et ont besoin d'ingénieurs polyvalents, formés à l'économie, la gestion du personnel autant qu'aux sciences de l'ingénieur ${ }^{24}$. Le secteur industriel grenoblois, relativement homogène, est alors dominé par l'hydraulique, la métallurgie, l'électricité et dans une moindre mesure la chimie.

Le cas de la section d'hydraulique illustre bien les relations évoquées cidessus. Durant les années 1950, les besoins en ingénieurs hydrauliciens changent, car les constructions de barrages hydroélectriques en France prennent fin. Les débouchés professionnels deviennent plus aléatoires. Le marché est en transition et l'école doit suivre ces évolutions. Tenir compte de l'évolution des besoins présente certaines difficultés : les enseignements doivent-ils être

21 Ibid.

22 Dominique Pestre, Louis Néel, le magnétisme et Grenoble, op. cit., p. 130-133.

23 Archives départementales [désormais $\mathrm{AD}$ ] de l'Isère, 8116W : Procès-verbaux des conseils de perfectionnement et conseils scientifiques de l'INPG.

24 AD Isère, 8116W97 : Conseil de perfectionnement du 7 juillet 1958. 
adaptés? Les sections doivent-elles changer de nom? Comment orienter les étudiants vers ces nouveaux besoins? Un représentant de l'association des anciens élèves, la Houille Blanche, s'exprime à ce sujet en 1957 :

"Certains hydrauliciens s'inquiètent. Lorsque fut créée l'École, son enseignement répondait à un besoin très net et indiscutable. Dans le domaine de l'hydroélectricité, il y avait beaucoup à faire. [...] Je crois que cette situation a complètement disparu; on peut dire que l'École a rempli une partie de son but et l'on se demande si elle ne doit pas s'adapter aux nouvelles utilisations de l'eau. Le règne de l'hydroélectricité n'est pas fini, mais on voit le terme des exploitations. Reste l'hydraulique agricole, reste l'hydraulique fluviale ou l'hydraulique des eaux des grandes villes, reste l'hydraulique à l'étranger. Il y a donc beaucoup de débouchés, mais ne serait-il pas bon de prévoir l'évolution et de faire une consultation des grandes administrations et des industriels, sur les besoins en ce domaine? ${ }^{25}$.

Au milieu des années 1960, le conseil de perfectionnement décide finalement que les hydrauliciens de Grenoble ne devront plus être considérés comme spécialisés uniquement dans la production d'énergie. L'hydroélectricité cède progressivement la place à "l'hydraulique à l'étranger" (irrigation, barrages, hydraulique urbaine) et à la mécanique des fluides. La modification progressive du marché de l'emploi impose une redéfinition des savoirs de l'ingénieur hydraulicien. La section connaît alors les mêmes difficultés que l'industrie hydraulique et doit modifier ses enseignements au gré des transformations du secteur.

Créée en juin 1955, la section de génie atomique connait un parcours un peu différent en suivant une logique plus "disciplinaire" qu'industrielle. L'acquisition du réacteur de recherche Mélusine et la fondation du Centre d'études nucléaires de Grenoble (CENG), dont la construction démarre quelques mois plus tard, sont guidées par les ambitions scientifiques des chercheurs grenoblois plus que par un besoin des industriels (même si dans les faits, l'implantation du CEA aura un rôle fondamental pour toute l'industrie grenobloise des matériaux) ${ }^{26}$. À ce titre, le poids des enjeux scientifiques dans la création de cette section préfigure les évolutions à venir. Quant à la section d'électrotechnique, elle est depuis sa fondation liée à la présence d'industries d'équipements électriques. Enfin, une section de mathématiques appliquées est organisée au début des

25 AD Isère, 8116W96 : Conseil de perfectionnement du 26 janvier 1957.

26 AD Isère, 8116W96 : Conseil de perfectionnement du 11 juin 1955. La création de la section va même être utilisée par Louis Néel pour obtenir l'installation du premier réacteur : voir Dominique Pestre, Louis Néel, le magnétisme et Grenoble, op. cit. 
années 1960. Sa mise en place se fait toutefois difficilement. La discipline peine en effet à trouver sa justification au sein de l'Institut. Une fraction importante des professeurs ne la considère pas comme un élément nécessaire au développement de l'Institut, car elle n'apparaît pas clairement, selon eux, comme un secteur industriel à part entière. En revanche, contrairement à ces derniers, les industriels grenoblois en perçoivent les atouts et, disposant d'une capacité à infléchir la politique de formation de l'Institut, contribuent à son institutionnalisation.

\section{Une recherche dispersée et connectée au monde industriel}

Au milieu des années 1950, cinq laboratoires sont rattachés à l'Institut. Parmi ceux-ci, le Laboratoire des essais mécaniques et de physique des métaux, chaux et ciments donne une bonne idée de la recherche qui a lieu à l'Institut à cette époque. Il s'agit à la fois d'un laboratoire de travaux pratiques destiné aux élèves ingénieurs et d'un laboratoire de recherche ${ }^{27}$. Les travaux pratiques sont financés par les fonds propres de l'Institut, mais les crédits s'avèrent insuffisants dans la mesure où les machines d'essais du laboratoire sont très anciennes, au point "que seul un entretien soigné et onéreux permet de les faire tourner encore ${ }^{28}$. En tant que laboratoire de recherche, quelques professeurs de l'Institut et techniciens du CNRS y étudient principalement les propriétés mécaniques physiques et thermiques des matériaux ainsi que leurs constitutions et structures. Ils y recherchent des "solutions de problèmes posés par les industriels et les services publics de l'État, de la région [et] de Grenoble ${ }^{29}$. Le laboratoire accueille quatre chercheurs dont, à titre d'exemple, les principales publications de l'année 1954 portent sur la durée de vie des ressorts, les méthodes d'usure des roches et les mesures d'écrouissage - durcissement après une déformation - des tôles. La recherche y est essentiellement financée par les industriels par le biais de la Société des amis du laboratoire, qui

$27 \mathrm{AD}$ Isère, 8116W26 : Lettre du ministère de l'Éducation nationale aux Recteurs d'académie du 12 décembre 1953.

28 AD Isère, 8116W26 : Laboratoire de physique industrielle et électrotechnique : année scolaire 19581959, «demande de financement exceptionnelle pour renouvellement du matériel du laboratoire de travaux pratiques".

$29 \mathrm{AD}$ Isère, 8116W26 : Note "Laboratoires de recherche (essais mécaniques et physique des métaux, chaux et ciment) : exercice 1954" du 15 mars 1959. 
s'ouvrira à d'autres laboratoires en 1958 pour devenir l'ADR, l'Association pour le développement des recherches ${ }^{30}$.

De son côté, le Laboratoire de physique industrielle développe des recherches qui portent essentiellement sur des questions liées à l'aimantation, les tôles métalliques, l'influence des chocs sur les machines électriques, les échangeurs de températures ou encore l'automatisation pour le génie chimique. Georges Kamarinos, directeur du Laboratoire de physique des composants à semiconducteurs dans les années 1980, raconte son arrivée dans un autre des laboratoires de l'Institut, le laboratoire d'électronique :

"Je suis arrivé [dans les années 1960] dans un petit labo où il y avait une dispersion des sujets scientifiques. Moi j'entrais avec un sujet à vocation électronique, d'autres faisaient de la physique pure et dure, d'autres faisaient des circuits électroniques, d'autres prenaient les réponses neuronales de la sciatique du lapin pour voir si on pouvait les reconduire avec un circuit équivalent. On n'était pas nombreux, vingt personnes en tout ${ }^{31}$.

La recherche est alors, au sein de l'Institut, une activité secondaire au regard des enseignements et des travaux pratiques. Elle est relativement dispersée et porte essentiellement sur des problèmes techniques très concrets que se posent les industriels grenoblois. La recherche académique a, quant à elle, essentiellement lieu au sein de la faculté des sciences, y compris lorsqu'elle est menée par les cadres de l'Institut. Maurice Fallot, directeur adjoint de l'Institut dans les années 1960, et René Pauthenet, directeur de l'École nationale supérieure d'ingénieurs électriciens de Grenoble au début des années 1970, travaillent au LEPM dirigé par Louis Néel. Julien Kravtchenko est à la fois professeur au sein de la section d'hydraulique et directeur du laboratoire de mécanique des fluides de la faculté. Pour l'électronique, les équipes, relativement réduites, de Jean Benoît et de Maurice Buyle-Bodin, directeurs successifs de l'école nationale supérieure d'électronique et de radioélectricité de Grenoble entre 1965 et 1983, sont sous la double tutelle de l'Institut et de la faculté des sciences.

30 Dominique Pestre, Louis Néel, le magnétisme et Grenoble, op. cit., p. 81-87.

31 Témoignage de Georges Kamarinos, dans Éric Robert, L’ingénieur, moteur de l'innovation, op. cit., p. 245. 


\section{La réforme Edgar Faure et l'autonomisation de l'Institut vis-à-vis de la faculté des sciences}

À la suite des événements de mai 68, la loi Edgar Faure est promulguée le 12 novembre 1968. La réforme instaure les unités d'enseignement et de recherche (UER), qui structurent désormais les universités ${ }^{32}$. À ce moment, l'Institut dispose encore du statut d'ENSI obtenu en 1948. À ce titre, il est une composante de l'université de Grenoble. Néanmoins, le conseil d'université ne prend jamais part à la définition des orientations stratégiques ni aux décisions concernant l'Institut qui jouit, de fait, d'une grande autonomie ${ }^{33}$.

Selon la loi du 12 novembre, les ENSI doivent devenir des UER, au même titre que les facultés. Cependant, l'Institut peut obtenir une série de dérogations lui permettant de conserver son identité : c'est-à-dire son mode de recrutement, son organisation administrative, une certaine liberté pédagogique et surtout, une autonomie en matière budgétaire. Mais son directeur de l'époque, Louis Néel, cherche une solution qui lui permettrait de sortir l'Institut du giron de l'université. Il est possible d'ériger l'Institut en "établissement public à caractère scientifique et culturel", mais cela ne lui semble pas suffisant, car ce statut ne prévoit pas d'autonomie vis-à-vis de l'université. Louis Néel précise son point de vue lors du conseil de perfectionnement du 7 décembre 1968 :

"Monsieur le ministre de l'Éducation nationale a d'ailleurs reconnu [le] caractère [particulier des ENSI] au cours d'une réunion récente des directeurs des ENSI. Il a reconnu que ces écoles, tout au moins les plus importantes d'entre elles, devaient être assimilées aux Grandes écoles. Les Grandes écoles, dans le langage du ministre ce sont : l'École polytechnique, l'École centrale, l'École supérieure d'électricité, etc. Pour elles, on envisage leur rattachement direct au ministère de l'Éducation nationale, sans interposition des nouvelles universités " ${ }^{34}$.

Localement, les arguments que Louis Néel avance devant le conseil de perfectionnement renvoient à la lourdeur administrative du fonctionnement universitaire, à la difficulté de maintenir un recrutement des élèves sur concours au sein de celle-ci et à l'intérêt pour l'Institut de conserver ses relations étroites avec le monde industriel grenoblois (souvent rétif à travailler avec les universités).

32 Christine Musselin, La longue marche des universités françaises, Paris, Presses universitaires de France, 2001, p. 57.

33 AD Isère, 8116W99 : Conseil de perfectionnement du 7 décembre 1968.

34 AD Isère, 8116W99 : Conseil de perfectionnement du 7 décembre 1968. 
Il est intéressant de noter qu'on retrouve des arguments similaires à la même époque dans le cas de l'Institut national polytechnique de Lorraine ${ }^{35}$. Les industriels locaux, par l'intermédiaire de Paul-Louis Merlin, de l'entreprise familiale Merlin-Gerin, soutiennent le projet de Louis Néel et rappellent leur défiance vis-à-vis du monde universitaire :

«En tant que chef d'entreprise, je voudrais vous dire que nous sommes très satisfaits des grandes écoles. Nous travaillons en collaboration avec l'Institut et les grandes écoles et l'on nous écoute lorsque nous disons qu'il y a une évolution que nous voudrions bien leur voir prendre. Je constate que les conseils d'administration des Facultés sont composés de jeunes gens qui n'ont aucune espèce d'expérience et de professeurs. Je ne vois pas pourquoi ces conseils d'administration ne comportent pas de délégués des grandes administrations, des délégués des grandes entreprises de façon à ce que véritablement le travail soit efficace ${ }^{36}$.

La plupart des membres du conseil de perfectionnement se positionnent en faveur de la proposition de Louis Néel. Seuls deux membres votent pour le maintien du rattachement à l'université. Jean Kuntzmann, le directeur des mathématiques appliquées, soutient ce rattachement au motif qu'il permettra de rapprocher la formation des ingénieurs de celle des techniciens.

Louis Néel construit son projet en lien avec Léopold Escande, professeur de physique à la faculté des sciences de Toulouse, et Marcel Roubault, professeur de géologie à la faculté des sciences de Nancy, qui sont, comme lui, membre de l'Académie des sciences ${ }^{37}$. Trois INP (pour Institut national polytechnique) sont créés par décret le 14 octobre 1969 (décret n69-930) à Grenoble, Toulouse et Nancy. L'Institut devient l'Institut national polytechnique de Grenoble (INPG) et rassemble trois écoles nationales supérieures d'ingénieurs (ENS). Quelques années et réorganisations institutionnelles plus tard, en 1974, il en comporte sept : l'ENS d'ingénieurs électriciens (ENSIEG), l'ENS d'hydraulique et de mécanique (ENSHMG), l'ENS d'informatique et de mathématiques appliquées (ENSIMAG), l'ENS d'électronique et de radioélectricité de Grenoble (ENSERG),

35 Françoise Birck, «Introduction : la Lorraine et ses enseignements techniques supérieurs", art. cit., p. 30; Michel Lucius, "Les origines et l'évolution de l'Institut polytechnique de Lorraine", in Françoise Birck, André Grelon (dir.), Des ingénieurs pour la Lorraine..., op. cit., p. 315-327 (entretien avec André Grelon).

36 AD Isère, 8116W99 : Conseil de perfectionnement du 7 décembre 1968.

37 Françoise Birck, L'école des mines de Nancy (ENSMN) 1919-2012 : entre université, grand corps d'État et industrie, Nancy, Presses universitaires de Nancy/Éditions universitaires de Lorraine, 2013, p. 233-234. 
l'ENS d'électrochimie et d'électrométallurgie de Grenoble (ENSEEG), l'École française de papeterie de Grenoble (EFPG).

Enfin, en 1968, le conseil de perfectionnement de l'Institut est dissous au profit de commissions consultatives d'enseignement internes à chaque école. Parallèlement, un conseil scientifique est créé dans le but de traiter les questions relatives à la politique scientifique de l'Institut. Étonnamment, les nouveaux statuts de l'Institut ne prévoient la participation de membres extérieurs ni au conseil scientifique ni dans les commissions d'enseignement (ce qui sera corrigé milieu des années 1980 pour le premier) ${ }^{38}$. Si le conseil de perfectionnement et le conseil scientifique n'ont en théorie pas les mêmes prérogatives, l'examen des archives montre qu'une continuité de fait s'est instaurée. Il s'agit de l'organe de discussion des politiques et des stratégies de l'établissement dont la fonction est d'orienter les décisions du conseil d'administration. Toutefois, la coopération avec les industriels, qui est l'argument fort en faveur de l'autonomie institutionnelle, va évoluer de manière tout à fait inattendue. Au moment où les membres de l'Institut réaffirment l'étroite relation entre la formation des ingénieurs et le monde industriel, les modalités mêmes de cette relation vont se transformer en profondeur.

\section{La transformation de l'Institut en institution de recherche}

\section{La déconnexion entre le monde industriel et la formation d'ingénieurs}

Le conseil de perfectionnement réunit des membres en fonction au sein de l'Institut et des membres extérieurs issus du monde académique et du monde économique (figure 1). Entre 1955 et 1968, il accueille en pratique entre 4 et 9 représentants industriels à chaque session (jusqu'à $40 \%$ des membres présents en 1959). Les représentants du monde économique sont issus soit des entreprises locales (Neyrpic, Merlin-Gerin, Sogreah, Association des producteurs des Alpes françaises), soit des grandes sociétés nationales (Compagnie nationale du Rhône, EDF, la SNCF, Société française des non-tissés). Les personnalités représentant l'Institut, quant à elles, sont les directeurs de ses différentes sections, certains de ses professeurs, ainsi que des chercheurs, professeurs et 
responsables académiques des autres facultés grenobloises. Le nombre de personnalités appartenant au monde académique oscille entre 7 et 13 personnes jusqu'en 1968. La composition du conseil scientifique mis en place en 1968 diffère quant à elle fortement de celle du conseil de perfectionnement qui lui précédait. D’un côté, comme nous l'avons signalé, les nouveaux statuts de l'Institut ne prévoient pas la participation de représentants du monde économique au conseil scientifique. Les statuts de l'INPG rétablissent la participation de membres extérieurs dans le courant des années 1980. Mais, le nombre de représentants effectivement présents reste très faible. Par ailleurs, lorsqu'ils sont présents, les représentants des sociétés locales des années 1960 ont laissé la place à ceux des groupes plus importants qui les contrôlent (Hewlett-Packard, Radiall ou ELF Solaize). D'un autre côté, le nombre de membres issus du milieu universitaire est multiplié par trois entre 1968 et 1988, les professeurs et les chercheurs de l'Institut étant les plus nombreux.

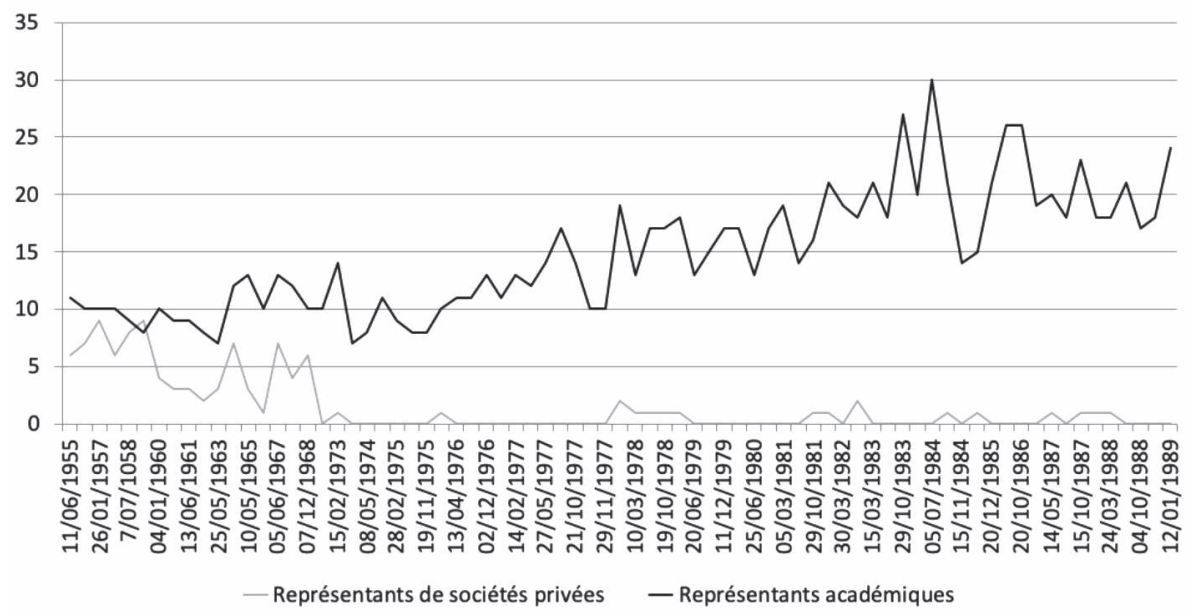

Fig.1 : évolution de la composition du conseil scientifique (1955-1989)

Les représentants des élèves et des services généraux de l'Institut ne sont pas comptabilisés. Sources : AD Isère, 8116W94-99 : Conseil d'administration de l'IPG, 1955-1968; AD Isère, 8116W282-289 : Conseil scientifique de l'INPG, 1971-1991.

Les nouveaux statuts de 1968 maintiennent toutefois la participation de représentants industriels au sein du conseil d'administration. Les industriels présents après 1968 sont d'ailleurs ceux qui assistaient au conseil de perfectionnement et au conseil d'administration avant la réforme : Henri Dagallier pour Neyrpic, Paul-Louis Merlin pour Merlin-Gerin, Banal pour EDF ou encore 
Bayard pour la Compagnie nationale du Rhône. Mais durant les années 1970, ces derniers sont progressivement remplacés par de nouveaux représentants dont la présence n'est pas régulière ${ }^{39}$.

Ce désengagement progressif des industriels des instances de pilotage de l'Institut ne manque pas de surprendre dans la mesure où le maintien d'une instance analogue au conseil de perfectionnement constituait l'un des principaux arguments de Louis Néel en faveur de la création de l'Institut comme université technologique autonome. Trois éléments permettent d'expliquer ce phénomène. Premièrement, les évolutions structurelles que connaît le milieu industriel grenoblois pèsent sur ses relations avec l'Institut. Les entreprises issues de la "seconde industrialisation grenobloise" ${ }^{40}$, telles que la chimie, la cimenterie, la métallurgie ou la papeterie, rencontrent des difficultés durant les années 1970. Dès le milieu des années 1950, les sociétés papetières grenobloises sont contraintes de rejoindre de grands groupements comme Arjomari. La société de construction de matériel électrique Merlin-Gerin est progressivement intégrée au groupe Schneider ${ }^{41}$. La société de construction de matériel hydraulique Bouchayer \& Viallet est fermée en 1971. Quant à sa consœur Neyrpic, elle devient une filiale du groupe Alsthom. Comme l'écrit Anne Dalmasso à son propos :

"Il s'agit là d'un exemple emblématique du changement d'échelle des stratégies : jusqu'aux années soixante, c'est au niveau local que sont conduites les politiques de développement par l'innovation et la diversification des activités, avec comme objectif de pérenniser le site. À partir des années soixante-dix, les mêmes stratégies sont menées à l'échelle du groupe, l'objectif est la pérennité de l'ensemble et non de chacune de ses parties " ${ }^{42}$.

Parallèlement, le secteur des petites entreprises technologiques prend un certain essor. À partir du milieu des années 1970, la première vague de création de petites entreprises technologiques est à l'initiative d'une cinquantaine d'ingénieurs issus de la SEMS-Télémécanique. Seize entreprises sont créées et installées sur la nouvelle Zone pour l'innovation et la recherche scientifique

39 AD Isère, 8116W72-75 : Conseil d'administration de l'INPG, 1971-1976; AD Isère, 8116W98-99 : Conseil d'administration de l'IPG, 1965-1968.

40 Henri Morsel, Jean-François Parent, Les industries de la région grenobloise, op. cit., p. 101.

41 Anne Dalmasso, "Vieilles et nouvelles technologies face aux mutations des années 1970 : l'Isère et le bassin grenoblois (1974/1984)", in Pierre Lamard, Nicolas Stoskopf (dir.), Une décennie de désindustrialisation (1974-1984)? Journées d'histoire industrielle, Paris, Éditions A. et J. Picard, 2009, p. 115.

Ibid. 
et technologique (ZIRST), située sur la commune limitrophe de Saint-Martind'Hères ${ }^{43}$. Ce milieu se caractérise par de petites PME- souvent sous-traitantes de grands groupes de l'électronique ou de l'informatique - dont la durée d'existence est assez courte ${ }^{44}$. Les décennies 1960 et 1970 correspondent à une transformation importante du tissu industriel grenoblois. Les sociétés grenobloises sont sous-traitantes, progressivement éliminées ou rachetées par de grands groupes nationaux et internationaux. Cette mutation du milieu industriel tend à diminuer le pouvoir de décision des industries locales en termes stratégiques. Elle donne ainsi une clé pour comprendre comment la relation entre les industries locales et l'Institut tendent à se transformer entre 1968 et le début des années 1980.

Deuxièmement, l'omission de représentants du monde économique coïncide avec la modification de la structure du tissu industriel évoquée précédemment. L'historien Hervé Joly explique qu'à partir des années 1970, "Le temps des notables polyvalents qui multipliaient les engagements dans plusieurs entreprises et dans d'autres sphères sociales et dont la notoriété n'était pas associée à une fonction précise est révolu ${ }^{45}$. La disparition de la figure du notable local et le renouvellement sociologique du patronat grenoblois contribuent probablement à ce désintérêt des industriels vis-à-vis du pilotage de l'Institut. Troisièmement, d'autres modes de relation sont en train de se mettre en place autour des collaborations de recherche et des partenariats avec les laboratoires eux-mêmes, notamment autour de structures pluridisciplinaires de recherche appelées groupements d'intérêt scientifique (GIS).

\section{Naissance d'une politique scientifique}

Comme le note Dominique Pestre, à compter des années 1970, "La recherche n'est plus ce à quoi les savants peuvent s'adonner lorsqu'ils n'enseignent pas, elle devient leur véritable raison d'être, la seule dimension intéressante et importante de leur travail $»^{46}$. En 1958, la Délégation générale à la recherche scientifique et technique (DGRST) est créée dans le but d'identifier des domaines scientifiques et techniques stratégiques et de les financer à partir d'appel d'offres. Elle doit

43 Thierry Bruhat (dir.), Vingt technopoles, un premier bilan : étude à l'attention de la DATAR, Paris, La Documentation française, 1990, p. 24.

44 Anne Dalmasso, "Vieilles et nouvelles technologies...", art. cit., p. 118.

45 Hervé Joly, "Les dirigeants des grandes entreprises industrielles françaises au XXe siècle : des notables aux gestionnaires", Vingtième siècle, vol. 114, n² 2, 2012, p. 31 .

46 Dominique Pestre, "La reconstruction des sciences physiques en France après la Seconde Guerre mondiale. Des réponses multiples à une crise d'identitén, Réseaux, hors-série 14, nº 1, 1996, p. 41. 
également «identifier les domaines dignes d'être créés (comme la biologie moléculaire) ou développés (comme la science des matériaux) ${ }^{47}$. Les questions relatives à l'implication économique des activités scientifiques et aux collaborations entre la recherche académique et l'industrie se font de plus en plus pressantes. En 1967, l'Agence nationale pour la valorisation de la recherche (ANVAR) est créée dans le but de valoriser et financer le transfert des connaissances auprès du CNRS, puis des universités et de tous les organismes de recherches ${ }^{48}$. La loi Edgar Faure enjoint les établissements à établir des partenariats avec des acteurs industriels locaux tandis que les aspects stratégiques de la recherche scientifique pour le développement économique prennent place dans les discours officiels $^{49}$. Plus tard, la loi du 26 janvier 1984 sur l'enseignement supérieur (dite "loi Savary") contribue à ce renforcement de la recherche au sein des institutions d'enseignement supérieur en faisant des activités de recherche une composante essentielle de la carrière des enseignants-chercheurs.

C'est dans ce contexte, qu'au milieu des années 1970, le conseil scientifique décide de doter l'Institut d'un document de cadrage intitulé "Note de politique scientifique ${ }^{50}$. Rédigée par le directeur de l'ENS d'électrotechnique et de génie physique, René Pauthenet, probablement à la demande du conseil scientifique, cette politique est explicitement orientée vers le secteur économique et structurée par trois grands axes thématiques : l'énergie, les matériaux et le traitement de l'information. Ce projet va contribuer à faire de la recherche le véritable moteur du développement de l'Institut. La mise en place de cette politique scientifique vise essentiellement l'obtention et la répartition des financements les plus importants délivrés par le ministère. Comme l'exprime le nouveau président de l'Institut, Philippe Traynard, au conseil scientifique :

"il faut être logique, si l'on fait progresser les trois axes à la fois, nous aurons trop de demandes et ce sera le saupoudrage. Au contraire, nous avons l'occasion de faire avancer un secteur et d'avoir une action plus prononcée ${ }^{51}$.

47 Ibid., p. 42.

48 Laure Reinhart, "De l'ANVAR à BpiFrance, en passant par Oséo : les grandes étapes du financement public de l'innovation", Annales des Mines - Réalités industrielles", n 1, 2014, p. 47.

49 Jérôme Aust, "Quand l'université s'ancre au territoire. Collaborations académiques et territoriales? Lyon (1958-2009)", Le Mouvement social, vol. 233, n²4, 2010, p. 107-125.

$50 \mathrm{AD}$ Isère, 8116W283 : "Note sur la politique scientifique de l'Institut national polytechnique de Grenoble - programmes de recherche 1977", 21 décembre 1976.

51 AD Isère, 8116W282 : Conseil scientifique de l'INPG du 9 décembre 1976. 
Les documents d'archives donnent peu d'informations sur la répartition des dotations du ministère de l'Éducation nationale. Toutefois, il apparaît clairement dans les comptes rendus du conseil scientifique que la politique scientifique de l'Institut a vocation à rendre lisible la recherche locale pour le niveau décisionnaire basé à Paris.

La stratégie de développement de l'Institut s'appuie également sur une transformation progressive de l'organisation de la recherche. D'une part, la recherche est déterminée par ses modes de financement. Les fonds nécessaires deviennent de plus en plus importants. Le coût des instruments scientifiques implique la création d'un consortium des gros appareils qui permet d'en mutualiser l'utilisation ${ }^{52}$. Pour être financé, tout nouvel instrument doit être pertinent vis-à-vis de l'environnement scientifique grenoblois dans son ensemble afin d'assurer qu'il sera utilisé de façon optimale. Les instruments deviennent ainsi constitutifs de la communauté scientifique grenobloise. D'autre part, à partir des années 1970, la recherche à l'Institut passe du modèle de l'expertise et de la sous-traitance (incarné par le laboratoire d'essais) à un modèle contractuel plus durable et porteur d'une recherche sur le long terme tel que le permettent les GIS. Le premier GIS de l'Institut est consacré à la magnétodynamique des liquides métalliques. Il réunit une équipe de thermodynamique et une équipe d'hydraulique autour d'un même sujet identifié comme stratégique. Comme le souligne René Moreau, professeur d'hydraulique, au conseil scientifique en 1977 :

"Il me semble ressentir le besoin, non seulement de répondre à la demande des industriels, mais de développer une compétence assez ferme par les études fondamentales, en disposant de temps et de moyens importants, de façon à ce que l'on ne travaille pas à la petite semaine, où en 18 mois, il faut répondre à une question précise et ensuite arrêter. Notre ambition est à longue portée. Il s'agit d'établir une doctrine - il n'y en a pas encore - et de participer à la recherche internationale dans ce domaine ${ }^{53}$.

Les structures de type GIS contribuent à associer l'identité de l'Institut à la recherche. Le doyen Bonnier propose en 1977 une description de la recherche qui s'établit au sein de l'Institut :

"L'INP est apparu comme l'un des rares établissements capables de présenter un programme pluridisciplinaire très concentré et, à ce titre, apparaît

52 AD Isère, 8116W283 : Conseil scientifique de l'INPG du 27 mai 1977.

53 Ibid. 
aujourd'hui comme un établissement relativement exemplaire du point de vue de la recherche ${ }^{54}$.

Les structures de type GIS permettent également d'établir des partenariats avec de grands groupes industriels en mutualisant les financements et en créant des pôles d'expertises sur un thème donné. Elles contribuent à transformer les modalités de coopération entre l'Institut et les industriels, de la question de la formation des ingénieurs à celle des partenariats de recherche.

Depuis la rédaction de la "Note sur la politique scientifique" du 21 décembre 1976, l'une des lignes de force explicite de la politique scientifique de l'Institut est "d'assurer une liaison étroite entre la formation des élèves ingénieurs et la recherche $»^{55}$. Cette volonté s'appuie sur l'orientation de la recherche sur des thèmes propres aux sciences de l'ingénieur. La note énonce à ce titre que "pour que [les] élèves reçoivent une formation actualisée, il est nécessaire que nos enseignants effectuent eux-mêmes une recherche actualisée "56. L'adossement des enseignements à une recherche de pointe est présenté comme une nécessité pédagogique. L'argument avancé dans la note est celui d'un nécessaire " transfert de connaissances" des laboratoires de recherche vers les salles de cours et les laboratoires de travaux pratiques.

\section{Adosser la formation des ingénieurs à une recherche de pointe}

La recherche à l'Institut croit constamment depuis les années 1950. Alors que l'Institut accueillait 5 laboratoires en 1950, il soutient plus de 25 structures de recherche à partir des années 1990. L'évolution du nombre de chercheurs témoigne de la véritable explosion de la recherche à compter des années 1980 (figure 2). Ce phénomène n'est pas propre à Grenoble, mais témoigne pour l'Institut d'une stratégie qui place la recherche au cœur de sa politique de développement.

À compter des années 1970, la mise en place d'une véritable politique scientifique est la nouvelle clé de voûte de l'Institut. Le début des années 1980 marque également le début d'une croissance plus forte du nombre de ses élèves (figure 3). La taille de l'Institut elle-même a considérablement augmenté depuis les années 1950. À cette époque, celui-ci était constitué de trois sections et accueillait chaque année quelques centaines d'élèves. En 1990, il réunit 7 écoles

55 AD Isère, 8116W283 : "Note sur la politique scientifique de l'Institut national polytechnique de Grenoble - programmes de recherche 1977», 21 décembre 1976. 


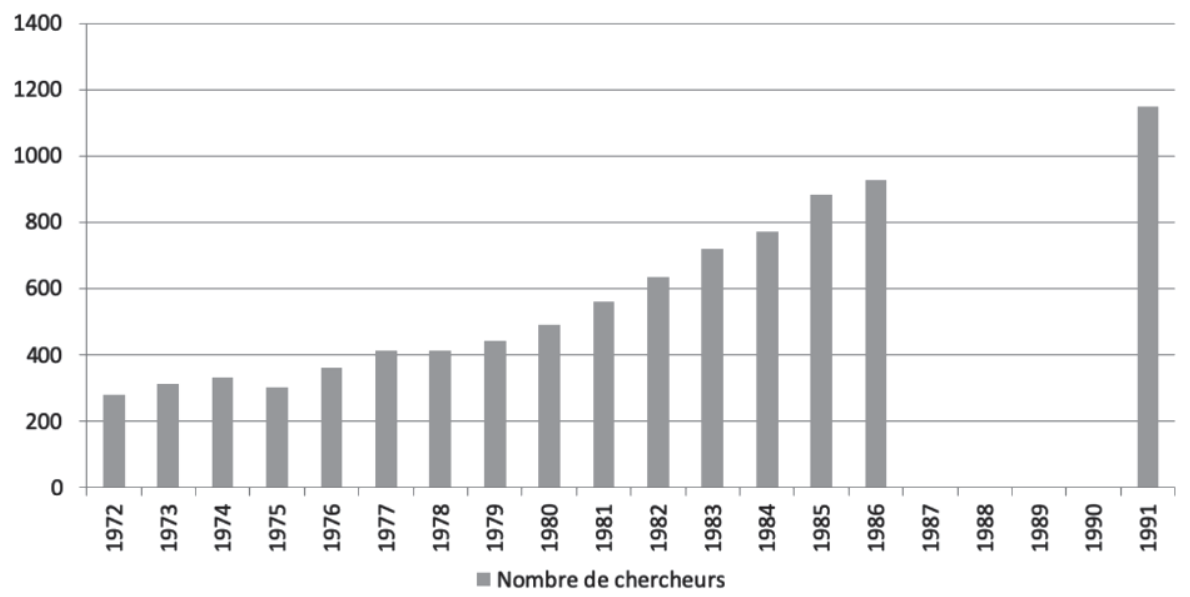

Fig. 2 : évolution du nombre de chercheurs à l'Institut entre 1972 et 1991

Source : AD Isère, 8116W251 (le graphique est obtenu à partir de la plaquette de l'INPG, "Institut national polytechnique de Grenoble", 1987, p. 15 et complété par 8116W289 : Georges Lespinard, "La politique scientifique de l'INPG", 1991).

et accueille plus de 2000 élèves. Là encore, cette augmentation spectaculaire n'est pas propre à l'Institut, mais participe d'un phénomène plus vaste de la croissance des effectifs d'étudiants dans l'enseignement supérieur ${ }^{57}$. Toutefois, la progression du nombre d'élèves ne dément en rien l'emprise grandissante de la recherche sur la formation des ingénieurs. Trois éléments peuvent être avancés en faveur de cette affirmation.

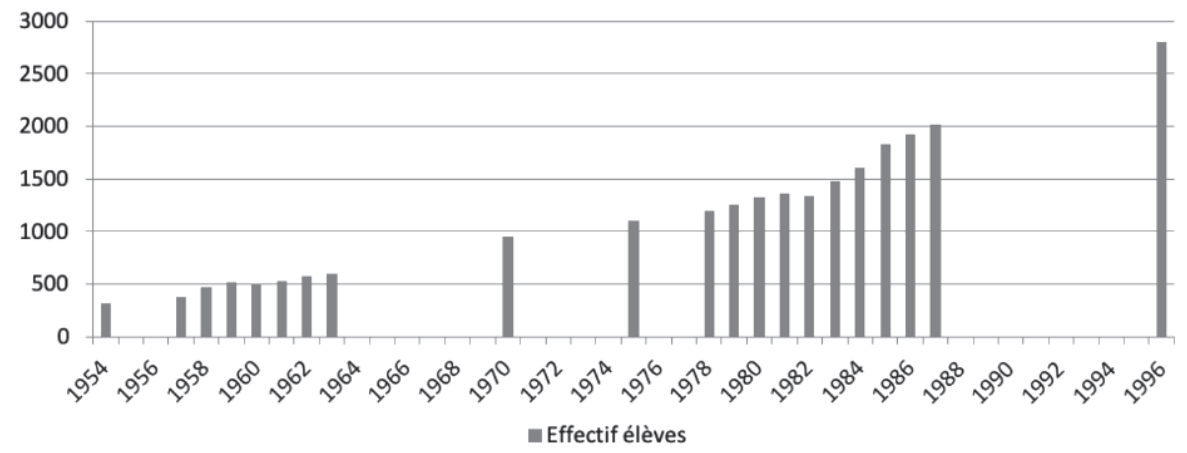

Fig. 3 : évolution des effectifs d'élèves entre 1954 et 1996

Source : AD Isère, $8116 \mathrm{~W}$ (données issues de divers documents des archives de l'Institut national polytechnique).

57 Christophe Charle, "Élites politiques et enseignement supérieur. Sociologie historique d'un divorce et d'un échec (1968-2012)", Pouvoirs, nº161, 2017, p. 31-50. 
Premièrement, le nombre de formations directement orientées vers la recherche augmente régulièrement. Entre 1979 et 1991, le nombre d'élèves inscrits en troisième cycle, qui mène au diplôme d'études approfondies (DEA) et au doctorat, a été multiplié par trois (figure 4$)^{58}$. S'ils ont principalement pour fonction d'ouvrir aux élèves la voie du doctorat, les DEA permettent également aux enseignants-chercheurs de repérer les meilleurs élèves, de les orienter vers la recherche et de les familiariser aux thèmes de recherches qui intéressent les laboratoires grenoblois. La "Note pour la politique scientifique" de 1976 stipule ainsi que la formation par la recherche représente "un avantage pour le recrutement des jeunes chercheurs dans [les] laboratoires " ${ }^{59}$ de l'Institut. Cet argument témoigne du renversement de l'ordre des priorités qui s'opère entre recherche et formation des ingénieurs. À ce titre, l'Institut accueille une cinquantaine de doctorants en 1976. Devenu une véritable institution de recherche en 1991, il en recense alors $650^{60}$.

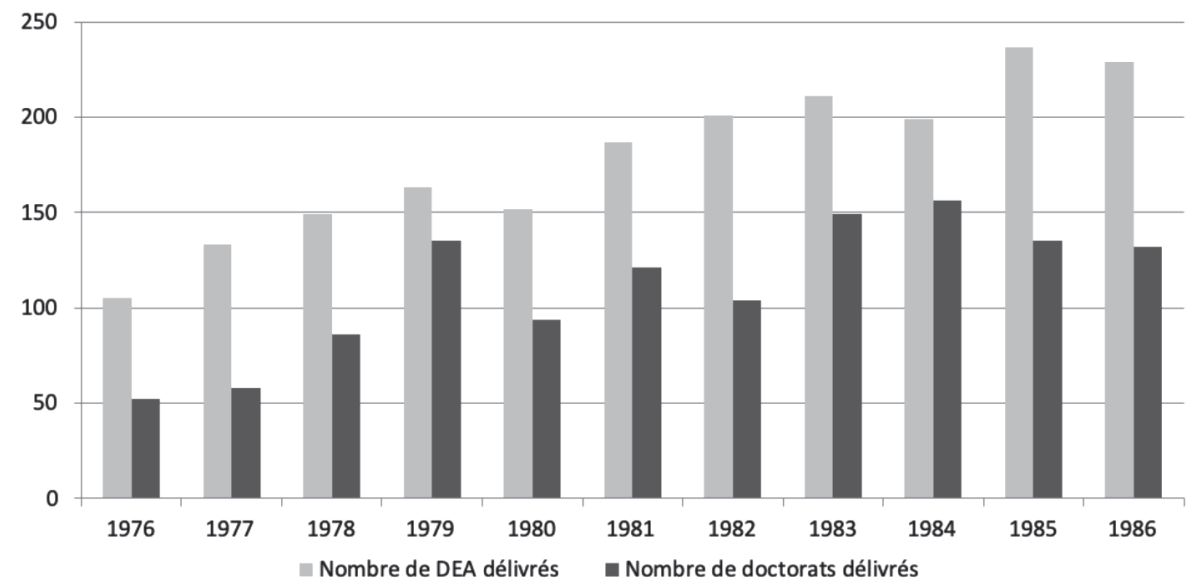

Fig. 4 : évolution du nombre de doctorats et DEA délivrés par l'Institut entre 1975 et 1986 Source : AD Isère, 8116 W251 (plaquette de l'INPG, "Institut national polytechnique de Grenoble», 1987, p. 30).

58 Commission nationale d'évaluation, L'Institut national polytechnique de Grenoble : rapport d'évaluation, 1991, p. 10

$59 \mathrm{AD}$ Isère, 8116W283 : "Note sur la politique scientifique de l'Institut national polytechnique de Grenoble - programmes de recherche 1977", 21 décembre 1976.

60 Agence d'évaluation de la recherche et de l'enseignement supérieur, Rapport d'évaluation de l'Institut polytechnique de Grenoble, décembre 2010, p. 5. 
À la fin des années 1980, sous l'impulsion de la Direction des enseignements supérieurs et de la recherche (DESR), l'Institut met en place des programmes de formation des ingénieurs "par la recherche" : les programmes FIRTECH (formation des ingénieurs par la recherche technologique), qui se déploient autour des thèmes de l'intelligence artificielle, de l'informatique et de la communication, des matériaux et de l'électronique ${ }^{61}$. L'objectif de la DESR est de doubler en cinq ans le nombre d'ingénieurs formés par la recherche afin de faire progresser la recherche technologique indispensable au développement de l'industrie française ${ }^{62}$. Les programmes FIRTECH regroupent plusieurs institutions et permettent d'apporter des financements complémentaires à certaines recherches intéressant les industriels. Le programme FIRTECH «Intelligence artificielle, informatique et communication", par exemple, regroupe l'École nationale supérieure de mathématiques appliquées de Grenoble (ENSIMAG), l'ENS d'électronique et de radioélectricité de Grenoble (ENSERG), les universités scientifiques de Grenoble et Chambéry ainsi que 9 laboratoires de recherche.

Deuxièmement, les ingénieurs de l'Institut trouvent de plus en plus de débouchés dans le secteur de la recherche publique. C'est le cas de l'ENSIMAG et de l'École nationale supérieure d'ingénieurs physiciens de Grenoble (ENSPG). Au début des années 1980, l'ENSIMAG envoie sur le marché de l'emploi environ 120 ingénieurs par an dont la moitié occupe des postes d'ingénieurs en informatique $^{63}$. Elle forme aussi beaucoup d'enseignants et de chercheurs universitaires ( $22 \%$ des élèves à la fin des années 1970). De son côté, entre 1975 et 1986, l'École nationale supérieure d'ingénieurs électriciens de Grenoble (ENSIEG) délivre 150 diplômes d'ingénieurs par an, dont la moitié trouve un emploi dans le secteur public (CNRS, CEA, ministère de l'Éducation nationale, de l'Enseignement supérieur et de la Recherche) ${ }^{64}$. Créée au sein de l'INPG en 1986 pour former des ingénieurs en électronique, en génie électrique et nucléaire, en matériaux et en instrumentation, quatre domaines correspondant

61 La question des FIRTECH aurait mérité un traitement plus systématique mais la dérogation pour consultation des archives portant sur ces programmes nous a été refusée.

62 AD Isère, 8116W251 : Plaquette de l'INPG, "Institut national polytechnique de Grenoble», 1987, p. 28.

63 AD Isère, $8116 \mathrm{~W} 280$ : "Votre salaire : Une enquête houille blanche menée par la commission placement au niveau national", octobre 1981.

$64 \mathrm{AD}$ Isère, 8116W280 : "Enquête portant sur les élèves des promotions 1975 à 1983 de la section de Génie-Physique de l'ENSIEG», s. d. [très probablement 1984]. 
"à des besoins industriels réels " ${ }^{65}$, l'École de physique (ENSPG) oriente quant à elle la plus forte proportion d'étudiants vers les laboratoires de recherche.

Troisièmement, certaines écoles de l'Institut revendiquent un enseignement fortement appuyé sur les laboratoires. Un rapport de 1991 du Comité national d'évaluation des établissements publics à caractère scientifique, culturel et professionnel soutient que "L'ENSIEG a réalisé un très bon couplage entre l'enseignement et la recherche" ${ }^{66}$ et que "la tonalité d'ensemble des enseignements [de l'ENSERG] est très orientée vers la recherche " $"{ }^{67}$. Un constat analogue est dressé pour l'école d'hydraulique, qui forme des ingénieurs pour de nombreux secteurs tels que le génie hydraulique, le génie civil, la mécanique, l'aérospatial ou encore l'automobile ${ }^{68}$. Le rapport d'évaluation indique à son propos que le "rayonnement de la recherche est considérable [...] mais [que] cette suprématie de la recherche n'écrase pas le développement des enseignements "69. Il est vrai que l'enseignement y est également adossé à l'Institut de mathématiques de Grenoble, qui permet de demeurer à la pointe des pratiques scientifiques avec l'usage de l'informatique et des méthodes numériques notamment. Au-delà de la formule, les auteurs du rapport relèvent que la recherche est au cœur de la mécanique grenobloise, et qu'elle guide la formation des ingénieurs.

\section{Conclusion}

L'avènement de la recherche à l'Institut national polytechnique de Grenoble se situe à l'intersection entre un contexte industriel local et des injonctions institutionnelles nationales qui constituent un jeu d'opportunités et de contraintes pour les acteurs locaux de l'enseignement supérieur et de la recherche. En pratique, l'histoire de l'Institut est le théâtre d'une lente "redéfinition de l'institution" entre la fin des années 1960 et le début des années 1980 ${ }^{70}$. D’une école d'ingénieurs portée sur l'environnement industriel local et national, l'Institut devient une institution de recherche orientée vers l'innovation et le transfert de connaissances. Les indicateurs de cette évolution sont nombreux et tous n'ont

\footnotetext{
65 Commission nationale d'évaluation, L'Institut national polytechnique de Grenoble, op. cit., p. 71.

66 Ibid., p. 29.

67 Ibid., p. 54.

68 Ibid., p. 16.

69 Ibid., p. 22.

70 Yves Gingras, "L'institutionnalisation de la recherche en milieu universitaire et ses effets", art. cit.
} 
pas pu être évoqués dans cet article : évolution du nombre de publications scientifiques, collaborations internationales, participation à de grands programmes de recherches, etc. Ce phénomène n'est pas propre à Grenoble. Ainsi, Armand Hatchuel signale un processus identique à celui que nous venons de décrire à propos de l'École des Mines de Paris lorsqu'il écrit que "l'école [des Mines] s'est métamorphosée en ce que l'on pourrait appeler un "institut des sciences pour l'ingénieur" " ${ }^{71}$. Ce processus d'institutionnalisation de la recherche est partie prenante d'un basculement plus général d'un "régime d'existence et de production des sciences " à un autre autour des années 1970 - pour reprendre la terminologie de Dominique Pestre ${ }^{72}$.

$\mathrm{Au}$ fond, cette institutionnalisation de la recherche combine deux renversements. Le premier consiste en un renversement des priorités de l'institution. La formation des ingénieurs n'est plus l'unique préoccupation des cadres de l'Institut. Pour être jugée pertinente, elle doit s'adosser à une recherche académique de pointe, connectée au monde industriel et aux réseaux scientifiques nationaux et internationaux. C'est la reconnaissance de cette activité de recherche qui fonde désormais la qualité de la formation des ingénieurs. Le second renversement qui caractérise ce processus d'institutionnalisation de la recherche est un changement d'échelle dans la définition des enjeux de l'institution. Si les cadres de l'Institut se saisissent de la réforme Edgar Faure pour affranchir l'Institut de la tutelle universitaire et se révèlent particulièrement entreprenants en matière de collaboration industrielle, cette institutionnalisation de la recherche répond également aux impulsions des politiques scientifiques nationales qui modifient les modalités de financement et d'organisation de la recherche. Certes, les enjeux économiques et technologiques locaux continuent d'orienter les choix stratégiques de l'Institut, mais c'est dans une configuration renouvelée dans laquelle la recherche est devenue le support principal des interactions avec les entreprises.

Thomas Lerosier

Université de Grenoble Alpes, Philosophie, pratique et langages (PPL) thomaslerosier@live.fr

71 Armand Hatchuel, "La naissance de l'ingénieur généraliste : l'exemple de l'École des Mines de Paris ", Réalités industrielles, 2006, p. 23.

72 Dominique Pestre, Science, argent et politique : un essai d'interprétation, Paris, INRA, 2003. 